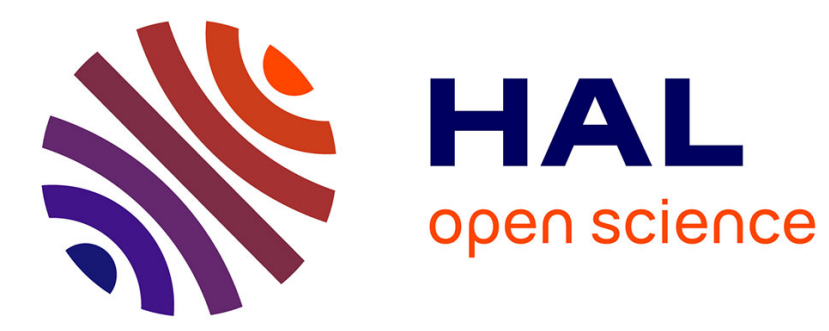

\title{
Mobiliser l'industrie de la chaussure, mobiliser ses territoires
}

\author{
Florent Le Bot, Cédric Perrin
}

\section{To cite this version:}

Florent Le Bot, Cédric Perrin. Mobiliser l'industrie de la chaussure, mobiliser ses territoires. Terrains et Travaux: Revue de Sciences Sociales, 2011, 2 (19), pp.205-224. 10.3917/tt.019.0205 . hal01470977

\section{HAL Id: hal-01470977 \\ https://hal.science/hal-01470977}

Submitted on 18 Feb 2017

HAL is a multi-disciplinary open access archive for the deposit and dissemination of scientific research documents, whether they are published or not. The documents may come from teaching and research institutions in France or abroad, or from public or private research centers.
L'archive ouverte pluridisciplinaire HAL, est destinée au dépôt et à la diffusion de documents scientifiques de niveau recherche, publiés ou non, émanant des établissements d'enseignement et de recherche français ou étrangers, des laboratoires publics ou privés. 


\section{Florent Le Bot, Cédric Perrin \\ Mobiliser l'industrie de la chaussure, mobiliser ses territoires}

\footnotetext{
$\mathbf{L}$
}

E TERRITOIRE est une construction sociale à laquelle l'État et les entreprises contribuent. L'échelle territoriale, nationale et locale, niveau de la coordination entre pouvoirs publics et entreprises, et la question de la nature de celle-ci, sont au cœur de nos préoccupations. L'industrie de la chaussure à travers sa configuration spécifique, organisée autour de trois formes de production (PME familiales isolées, mais surtout grandes entreprises intégrées et districts industriels) semble particulièrement se prêter à une telle étude, associant relative identité des marchés et diversité d'organisations. Cette notion de district industriel a le mérite d'éclairer des évolutions économiques à une échelle de coordination spécifique, longtemps mésestimées (Daumas, 2007 ; Zeitlin, 2007). Elle s'inscrit dans une historiographie renouvelée des PME (Odaka, Sawai, 1999 ; Guillaume, Lescure 2008), ainsi que dans une sociologie de l'action publique en matière territoriale (Wachter, 1989 ; Courlet, Soulage, 1995 ; Le Galès et alii, 2001, 2004 ; Le Galès, 2005) et une socioéconomie en plein essor et mobilisée dans une perspective historique (Zalio, 2005, 2006), qui doivent nous permettre d'enrichir ces analyses.

La problématique des districts industriels (Marshall, 1906) a resurgi au cœur des sciences sociales lorsque la crise économique des années 1970 et les décennies de dépression consécutives ont fait vaciller le modèle du gigantisme industriel fordiste (Bagnasco, 1977 ; Becattini, 1979). Les pouvoirs publics des pays développés, dont les français, ont cherché des alternatives à travers un intérêt renouvelé pour les PME et les territoires industrialisés dédiés à des productions spécifiques. Dans la longue durée, cette dimension n'est pas nouvelle, puisqu'elle vient s'inscrire dans l'histoire du rôle mobilisateur auquel s'astreint l'État durant le $\mathrm{XX}^{\mathrm{e}}$ siècle en 
matière économique et sociale. La question posée est celle des voies suivies par l'action publique en matière de mobilisation des ressources; le terrain privilégié, s'agissant de l'industrie de la chaussure, est celui des concentrations géographiques de PME (Fougères, Romans, Cholet, etc.), confrontées au développement de concentrations usinières et en groupes (Chaussures André, etc.). Nous montrerons que l'État dans sa recherche d'institutions partenaires, pour assurer sa prise sur l'économie, est dans l'obligation impérative de prendre en compte les reconfigurations du système productif, sans forcément y réussir.

Il s'agit de confronter l'histoire économique à une sociologie en renouvellement. Une sociologie de l'action publique s'est construite (essentiellement à partir des années 1990 en Europe, de manière plus ancienne aux États-Unis) en réaction à l'idée d'une sociologie du politique dont l'objet serait d'analyser l'État dans la seule perspective de son action verticale, hiérarchisée et descendante. Cette sociologie a pu émerger dans un contexte de segmentation de la décision (processus de décentralisation politique, intégration européenne, globalisation économique), encourageant à prendre en compte le pluralisme dans l'action collective, la pluralité des intervenants et des échelles d'interventions en matière de mobilisation des ressources. Cette évolution épistémologique invite à relire dans la durée l'action de l'État, entre autres en matière économique, dans ses rapports à une pluralité d'interlocuteurs ; d'un État "peu puissant" en quête de partenariats. Nous privilégions ici une catégorie d'interlocuteurs, les organisations patronales, dont les interventions, s'agissant de l'industrie de la chaussure, se jouent au niveau local, à travers des groupes de pression organisés dans le contexte des systèmes productifs localisés, mais aussi au niveau national dans des fédérations patronales, selon des intérêts parfois contradictoires ou divergents. Nous bénéficions d'une historiographie et d'une socioéconomie des mondes patronaux dynamique (Zalio, 2004 ; Daumas, 2010). La période retenue, 1914-2000, permet d'observer une variété de jeux d'agencements et de réagencements dans les rapports entre l'État et ses interlocuteurs patronaux.

La guerre de 1914-1918 et ses suites, la crise économique et les crises politiques des années 1930-1940, la période de croissance puis de dépression économique et de mutations du capitalisme des années 1950-2000, constituent autant de séquences au cours desquelles les politiques publiques s'essaient à un dosage différencié entre mobilisation économique verticale, s'appuyant sur les organisations patronales nationales, et prise en compte, à travers des politiques territoriales adaptées, des dynamiques propres aux PME et aux districts industriels. Les archives mobilisées per- 
mettent de croiser les échelles et les points de vue : archives d'entreprises, de groupements patronaux (chambres de commerce, chambres des métiers, syndicats professionnels, etc.), périodiques professionnels, archives bancaires (notamment du Crédit lyonnais pour les études sectorielles et de la Banque de France pour les études territoriales), archives publiques nationales (des ministères de la Production industrielle, de l'Économie, des Finances et de l'Industrie, du Commissariat général aux questions juives conservant un grand nombre de dossiers d'entreprises, etc.) et locales (archives départementales et municipales) (Le Bot, 2007 ; Perrin, 2007).

\section{La République radicale et les entrepreneurs (1917-1929)}

\section{Étienne Clémentel, la guerre et la mobilisation industrielle}

L'analyse de la Grande Guerre comme phase décisive pour l'intervention économique de l'État et la mise en place des prémices d'une organisation industrielle n'est pas neuve (Kuisel, 1984 ; Horn, 1997). Les besoins de l'effort de guerre commandent une mobilisation de l'économie, de la main d'œuvre, de la société dans son entier, tendue vers la victoire ; il s'agit également, dans la perspective d'un conflit installé dans la durée, d'assurer un équilibre social. P. Fridenson a souligné l'importance de la période pour l'acclimatation des méthodes tayloriennes en France et l'implication de l'État en la matière (Fridenson, 1987). Des travaux sont venus préciser le rôle de personnalités dont celle d'Étienne Clémentel qui a regroupé sous son autorité, d'octobre 1915 à novembre 1919, la plupart des ministères économiques (Druelle-Korn,2004). Radical, Clémentel a déjà exercé plusieurs mandats ministériels (les Colonies, l'Agriculture, les Finances) avant la guerre. Sa carrière politique, de tout premier plan (il est ministre des Finances du cartel des gauches en 1924-1925), s'étend jusqu'en 1935. À la fin de l'année 1916, alors que le prix d'une paire de chaussures a triplé depuis le début du conflit, Clémentel propose d'utiliser les surplus des stocks de cuir du programme de chaussures militaires pour mettre en fabrication une chaussure bon marché pour les classes populaires ${ }^{1}$. Le 14 août 1917, le Journal officiel publie le décret instituant la " chaussure nationale ». Dès le 22 septembre, les premières paires sont mises en vente dans la région de Toulouse. Il faut toutefois attendre décembre pour que leur vente se généralise. D’octobre 1917 à janvier 1918, leur production

1. Service historique de l'armée de terre, 10 N187, Commission d'enquête des marchés de l'État. Historique de la chaussure nationale, 1924. Godfrey (1987). 
passe de 30000 à plus de 500000 paires par mois. Malgré cette croissance, l'offre reste inférieure à la demande et ces chaussures sont difficiles à trouver. À la Chambre des députés, les socialistes accusent les fabricants de ne pas faire tous les efforts possibles afin de produire des quantités suffisantes. De fait, dès avril 1917, alors que le projet n'était encore qu'à l'étude, les milieux professionnels avaient fait part de leur opposition : "Nous faisons appel à toutes les chambres syndicales de la chaussure pour protester contre ce projet. Si le besoin s'en faisait sentir, que le gouvernement fasse fabriquer une certaine quantité de chaussures civiles en réquisition, mais qu'il laisse fabricants et détaillants pourvoir de leur mieux aux demandes de leurs clients. $»^{2}$ L'inquiétude est forte chez certains fabricants, particulièrement parisiens, de se voir imposer, outre la production de chaussures militaires, celle de chaussures uniformes pour les civils, au risque de voir la clientèle se tourner vers la concurrence étrangère, voire vers des centres de province moins concernés par ce programme. La configuration émiettée des places productives va contre le projet uniformisé de l'État. Le ministère du Commerce tente de rassurer les fabricants ; mais la volonté d'apaisement, se double d'une ferme menace de réquisition afin de soumettre les éventuels récalcitrants. La peur de désordres sociaux engendrés par une pénurie conduit les députés conservateurs à mettre entre parenthèses leur défense de la libre-entreprise et à ne pas s'opposer à l'intervention de l'État. En août 1918, la production atteint plus de 800000 paires, donnant raison à la ténacité de Clémentel, et le parlement reconduit le programme. Du $1^{\text {er }}$ septembre 1917 au 28 février 1919, près de 9,3 millions de paires sont produites et la production se poursuit jusqu'au mois de juillet 1919 . Ainsi, se dessine l'engrenage, sinon d'une " grande transformation » version Polanyi - l'État ne s'est pas abstenu d'intervenir dans l'économie au $\mathrm{XIX}^{\mathrm{e}}$ siècle - (Polanyi, 1983), du moins, plus simplement, d'une mobilisation croissante de l'économie par le politique.

\section{L'interlocuteur patronal : échelle nationale ou régionale?}

Les difficultés rencontrées dans le déploiement de ce programme, comme dans d'autres, conduisent Clémentel à envisager un renforcement de la coordination entre pouvoirs publics et patronat. Ainsi, appelle-t-il à « renverser les cloisons étanches qui pouvaient séparer à la fois le gouvernement, le parlement, l'administration, le commerce et l'industrie. La guerre nous a contraints à la collaboration. Nous avons vécu côte à côte,

2. Le Cuir, $1^{\text {er }}$ mai 1917. 
nous avons vécu ensemble, nous avons appris à comprendre les efforts réciproques que nous pouvions faire pour le bien de tous. C'est avec certitude que cette union sacrée, née de la guerre, se continuera, que nous devons multiplier nos efforts qui aboutiront, j'en suis persuadé, au succès et à la victoire économique de la France. ${ }^{3} \mathrm{Il}$ choisit le cadre de l'Association nationale d'expansion économique (ANEE) pour cette déclaration. L'ANEE, créée en 1915 avec le soutien de Clémentel, est une première ébauche avant la fondation de la Confédération générale de la production française (CGPF) en 1919, toujours avec le soutien du ministre. Il s'agit de renforcer la coordination entre l'État et l'industrie par l'institution d'interlocuteurs représentatifs.

Clémentel adopte une stratégie identique avec le monde de l'artisanat par l'intermédiaire de deux petits fabricants de chaussures parisiens, Georges Grandadam et Robert Tailledet. En 1919, alors que leur profession connaît des difficultés, les deux hommes réunissent deux organisations de cordonniers parisiens au sein d'un nouveau syndicat professionnel : la Fédération de la Petite Industrie de la Chaussure (FPIC), afin d'obtenir l'aide de l'État ${ }^{4}$. Clémentel accepte de les soutenir, dans la mesure où, comme le grand patronat avant eux, ils constituent une organisation rassemblant tous les artisans et pas seulement ceux de la chaussure : en 1922, voit le jour la Confédération Générale de l'Artisanat Français (CGAF), première organisation de l'artisanat en France. Celle-ci obtient de l'État toute une série de mesures qui concrétise l'existence de l'artisanat en lui offrant une reconnaissance publique (Perrin, 2007).

Toutefois, cela ne comble pas pleinement la volonté affirmée par Clémentel de prise sur les structures industrielles. Les pouvoirs publics doivent composer avec l'atomisation de la production. Il existe alors plusieurs dizaines de milliers de fabriques de chaussures dans le pays, la majorité petites, voire très petites. En même temps, la production est concentrée dans quelques localités, dont certaines (Fougères, Romans, etc.) présentent clairement les caractéristiques de systèmes productifs localisés (SPL), ou si l'on préfère de districts industriels marshalliens : accumulation dans le temps d'un savoir-faire, concurrence/coopération entre entreprises par la division du travail et le partage des marchés, machines-outils performantes proposées en location par la United shoe machinery corporation (USMC), circulation de l'information et de la main d'ouvre, circuit financier adapté, identité collective forte valant

3. Discours de Clémentel devant l'ANEE, printemps 1917, cité dans Kuisel (1984), p. 87.

4. Archives nationales (AN), F12 8807, Congrès national de la FPIC. Zarca (1986) ; Zdatny (1999). 


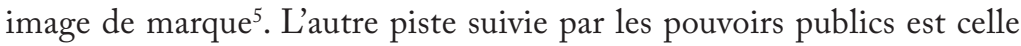
de la politique territoriale.

Parmi les tendances politiques à l'œuvre depuis l'avant-guerre, celle du député et négociant en cognac Jean Hennessy (député à partir de 1910, ministre de l'Agriculture de 1928 à 1930), prône un régionalisme reposant sur la légitimité des milieux professionnels : « la profession représentée dans la région organisée » (Dubasque, 2008 : 81). L'ambition est que ceux-ci s'emparent, dans leur bassin d'activité, des questions de développement économique. Toutefois, malgré de nombreux débats à la Chambre, la politique territoriale débouche uniquement sur la mise en place d'organes consultatifs : en 1915, les comités consultatifs d'action économique et en 1919, 17 groupements économiques régionaux s'appuyant sur 136 chambres de commerce, les « régions Clémentel ». L'inquiétude est grande pour une partie du personnel politique, de voir un régionalisme justifié à des fins d'optimisation des ressources, déboucher sur le fédéralisme honni depuis au moins $1793^{6}$. Les mesures prises par l'État pour mobiliser l'économie au-delà de la période de guerre, ne prennent qu'en partie la mesure des structures industrielles du pays. Cherchant à installer des interlocuteurs représentatifs, les pouvoirs publics favorisent l'organisation des milieux patronaux au niveau national. Ce faisant, ils minimisent le fait que l'économie française repose sur un tissu de PME pour beaucoup regroupées au sein de places productives localisées et que le niveau national n'est pas forcément le plus efficient pour les mobiliser. Mais il s'agit également, en appuyant de la sorte les classes moyennes indépendantes, d'assurer l'assise du régime selon la «synthèse républicaine » définie par Stanley Hoffmann ${ }^{7}$.

\section{La crise du pacte républicain (années 1930-1940)}

\section{Un patronat réactionnaire}

La crise des années 1930 vient bouleverser la donne. D'une part, l'émergence dans la filière de grands groupes intégrés (les français André, Pillot

5. S'agissant des débats autour du concept de « district industriel ", nous renvoyions à Le Bot, Perrin (2011). 6. Depuis l'épisode de la lutte opposant Montagnards centralisateurs et Girondins fédéralistes, débouchant notamment sur l'insurrection, encouragée par les seconds contre le pouvoir des premiers, d'une partie des départements français à l'été 1793 , le thème de la décentralisation a durablement été associé dans le débat politique, à une mise en cause de la République " une et indivisible ». Ozouf (2009).

7. Dans cette perspective, la stabilité de la République passe par une alliance objective entre le parti radical, qui y gagne des élus, et les classes moyennes indépendantes de l'industrie et du commerce qui obtiennent pour prix de leur soutien, des mesures législatives favorables. Sick (2003). 
et surtout le tchèque Bata) ébranle la position des districts. D'autre part, les pouvoirs publics ont désormais face à eux des communautés patronales organisées, localement et nationalement, pour faire valoir leurs revendications défensives, protectionnistes et malthusiennes, exprimées sur un mode réactionnaire (contre toutes les novations introduites par les grands groupes, censées " avilir la qualité » et " avilir les prix » et refléter des conceptions d'origine "étrangère » ou « juive »).

En février 1935, alors que depuis l'année précédente Bata dispose d'une usine dans l'Est de la France, les industriels fougerais créent un « comité de défense » et lancent un appel aux autres centres de production pour qu'ils s'organisent face à la concurrence étrangère ; concrètement face à Bata ${ }^{8}$. Dans la continuité, le député de Fougères, Étienne Le Poullen (conservateur, élu en 1933), dépose un projet de loi visant à protéger l'industrie de la chaussure.

Vers 1930, Fougères, ville moyenne du nord-est de la Bretagne, est quasi mono-industrielle, avec 92 fabriques de chaussures, un vivier de maind'œuvre de 7 à 8000 personnes pour une production annuelle de 4,5 millions de paires (près de $10 \%$ des salariés de la chaussure en France et environ $10 \%$ de la production, autant que le groupe André). À ces fabriques, s'ajoutent une trentaine d'entreprises complémentaires. Soulignons aussi la présence de 3 banques locales soutenues par une succursale de la Banque de France qui contribuent à la dynamique financière du SPL9 9

En 1935, il n'y a plus que 73 fabriques produisant seulement 2,5 millions de paires. La main-d'œuvre est réduite à 5000 personnes, avec un taux de chômage de 12 \%. La principale banque locale est liquidée en $1933^{10}$. Ce SPL n'apparaît plus en concordance avec les méthodes productives les plus modernes, celles notamment employées par Bata (chronométrage des tâches, travail à la chaîne, distribution intégrée) ; son produit, la chaussure pour femmes moyenne gamme, n'est plus autant désiré. Loffre fougeraise n'est plus adaptée aux besoins d'une clientèle paupérisée par la crise. Les prix pratiqués par la fabrique dominante (Morel \& Gâté) sont supérieurs de 15 à $25 \%$ à ceux des grands groupes ${ }^{11}$.

La protestation fougeraise gagne les autres centres et les comités anti-Bata multiplient leurs réunions à travers le pays. Les syndicats patronaux s'or-

8. Chambre de commerce et d'industrie de Paris (CCI), VI-1.20 (2), Industrie de la chaussure, 1935-1940. Le Bot (2005).

9. Archives de la Banque de France (ABdF), rapports d'inspection de la succursale de Fougères, 1932 et 1933.

10. Idem, 1935 et 1938.

11. Archives municipales de Fougères, 24Z 170-1391, Archives des établissements Morel et Gâté, 1902-1976. 
ganisent sur un mode plus offensif. En janvier 1935, le Syndicat général de la chaussure de France, jusque là peu représentatif, se transforme en Fédération nationale de l'industrie de la chaussure de France, c'est-à-dire une fédération de groupements professionnels régionaux (les adhésions individuelles nétant plus reçues qu'à titre exceptionnel). Ce faisant, il prend acte de l'organisation territorialisée de la production. Ce changement s'accompagne d'une croissance de plus de $25 \%$ de ses adhérents. Le syndicalisme patronal bénéficie des inquiétudes de ses mandants face à la crise économique et aux mutations de la branche.

Sous la pression, la loi Le Poullen, adoptée le 22 mars 1936, interdit l'ouverture de nouvelles fabriques de chaussures, magasins, rayons de vente ou organismes quelconques de réparation ou de distribution de chaussures au détail, ainsi que les transferts ou travaux d'agrandissement (sauf autorisation). En avril, la loi Paulin, voulue par la FPIC de Tailledet, interdit à tout non-artisan d'exploiter un atelier de réparation de chaussures. La succession des mesures législatives signale l'installation en position de force, au plan politique, des représentants des PME, en même temps que leur situation économique se dégrade en profondeur. Les pouvoirs publics qui ont établi la représentation patronale en interlocutrice représentative et qui mène une politique de protection des structures économiques à des fins de stabilisation sociale et politique, se trouvent dépassés par les revendications des centres de production locaux. La politique de prise sur l'économie n'a pas tenu compte de la réalité des districts industriels. Quand la crise en fait vaciller certains, l'État ne sait pas proposer une politique territoriale, et adopte des mesures protectionnistes sous les encouragements des syndicats patronaux.

\section{Vichy, temps béni des artisans?}

Le temps de la guerre est celui des illusions, des compromissions et des malentendus. Les artisans et les patrons de PME de la chaussure, du moins une large partie de ceux engagés dans la représentation professionnelle (d'autres sans doute aussi) accueillent favorablement le régime de Vichy. Ils se retrouvent dans sa vision du travail et de l'économie française. Pour les pétainistes, la France est un pays industriel par accident et l'industrialisation contraire à sa vocation de pays rural et de petits producteurs. Le travail par excellence est celui du paysan et de l'artisan (Perrin, 2008). La propagande de la Révolution nationale se gargarise de références à un passé préindustriel mythifié. Elle développe un discours antilibéral hostile à la grande entreprise. Le mouvement anti-Bata trouve en Vichy un aboutissement à son discours réactionnaire. Sa tonalité xénophobe s'ac- 
commode sans trop de difficultés de l'antisémitisme du régime. Pour les représentants patronaux du cuir, majoritairement à la tête de moyennes entreprises, "l'aryanisation économique " (le vol des entreprises dites « juives ») devrait pouvoir servir à la réorganisation du secteur par l'élimination des « trusts apatrides » et des artisans « d'importation $»^{12}$.

Du côté des organisations d'artisans, l'accueil positif aurait pu sembler moins évident. Certes, Robert Tailledet n'a eu de cesse durant la crise des années 1930 de pester contre le développement de la " camelote » pour expliquer les difficultés croissantes des artisans cordonniers et il s'est engagé dans la promotion de la loi Paulin censée protéger les petits ateliers ${ }^{13}$. Mais, il s'inscrit dans le courant radical-socialiste et avec la CGAF, au niveau de l'ensemble artisanal, il impose une conception classiste et syndicaliste - les artisans forment une classe sociale qui a ses propres intérêts qu'un syndicat doit défendre - contre les conceptions corporatistes. Or, Vichy est corporatiste. L'entente n'est donc pas a priori acquise. Néanmoins, Tailledet accepte les gages de bonne volonté du régime. Ce ralliement va dans un premier temps éviter à la CGAF d'être dissoutes comme les autres confédérations.

Toutefois, les relations se tendent rapidement entre Vichy et les artisans. Ceux-ci se montrent de plus en plus mécontents. Le problème vient de l'écart entre l'affichage et la réalité de la politique menée, beaucoup moins favorable aux petits entrepreneurs. Vichy n'est pas un bloc uni mais un amalgame de réactionnaires et de technocrates modernisateurs, traversé de tensions et de contradictions. Jusqu'en 1941, la Révolution Nationale paraît au premier plan. Ainsi, est créé un service de l'artisanat au sein du ministère de la Production industrielle, ce que même la III ${ }^{e}$ République radicale n'avait pas fait ${ }^{14}$. Ce service élabore des programmes de production spéciaux, qui, à l'inverse de ce qui s'était passé lors de la Grande Guerre, s'avèrent des échecs complets. Il promeut le « Retour à la Terre ", invitant les ouvriers des villes à s'installer comme artisans à la campagne. Ces actions dans l'esprit de la Révolution nationale, ne répondent pas aux problèmes concrets des petits producteurs, et d'abord à celui des pénuries. Les comités d'organisation mis en place en août 1940 afin d'organiser l'économie sur un mode corporatiste, s'enfoncent au fil de l'Occupation

12. Cf. par exemple, AN, AJ38 2192, rapport du vice-président de la Chambre syndicale du commerce de détail et de la maroquinerie et administrateur provisoire de la Sté de Maroquinerie Select au préfet de police de Paris, direction du contrôle des commissaires gérants, le 30 janvier 1941. Le Bot (2007).

13. TAILLEDET, R. (1937) : La doctrine de classe de l'artisanat moderne, Paris, Institut National des Métiers. 14. Les archives de ce service sont conservées aux AN, F12 10226 et suiv. 
dans une gestion de plus en plus contraignante de la pénurie (Joly, 2004). Les petites entreprises et plus encore les artisans y sont très mal représentés. La distribution de ressources, rares et recherchées, alimente un mécontentement croissant chez ceux-ci, dont témoigne un flot de protestations, accueilli par Vichy comme un travail de sape radical-socialiste. Pour y mettre fin, sans d'ailleurs totalement y réussir, la CGAF est dissoute en 1942. Après-guerre, les organisations artisanales ne se remettent pas de leur compromission initiale et les métiers de la chaussure ne retrouvent jamais au sein de l'artisanat, dont ils ne sont d'ailleurs plus qu'une branche secondaire et déclinante, l'influence qu'ils ont pu avoir dans l'entre-deuxguerres.

De fait, la période de Vichy a cristallisé les structures du secteur. Il n'y a pas eu réduction du nombre d'entreprises comme les technocrates rationalisateurs de Vichy le souhaitaient. "L'aryanisation économique » a éliminé, pour l'essentiel, de très petites entreprises, mais de nombreuses entreprises champignons surgissent pendant et après la guerre. Les grandes entreprises intégrées n'ont pas été démembrées, y compris par la voie de «l'aryanisation » (les projets de spoliation du groupe André n'ont pas été menés à leur terme ${ }^{15}$ ), même si leur capacité de production a été largement entravée (en particulier pour Bata). La reprise puis la croissance des années 1950-1960, leur permettent de retrouver leur dynamisme. Vichy n'est qu'une parenthèse pour certains districts (particulièrement Fougères) qui donnaient déjà des signes d'essoufflement dans les années 1930. Le retour à un fonctionnement normal du marché apparaît d'autant plus périlleux que ceux-ci n'ont pas évolué ; leurs entreprises s'avèrent vieillissantes et mal adaptées aux nouveaux besoins de la consommation.

Autre limite : Vichy est un État affaibli par l'Occupation, mais c'est un État autoritaire et bureaucratique, comme l'illustre la rupture avec la CGAF. Les PME ont pu profiter de cette période, mais en dehors de toute véritable politique industrielle construite pour elles et autour des districts. La création des préfets régionaux en 1941, assistés notamment d'un Intendant des affaires économiques, ne change rien en la matière, leurs interventions étant dominées par les problèmes de ravitaillement.

15. Les deux propriétaires du groupe André étant considérés comme " juifs " du fait de la "législation " antisémite de Vichy et de l'Occupant allemand, le groupe est placé sous administration provisoire (AP) dès novembre 1940 afin d'être vendu. Les convoitises sont fortes autour d'un groupe qui fournit à l'époque environ $10 \%$ des chaussures françaises et qui dispose d'un outil de production et de commercialisation parmi les plus modernes. Devant l'impossibilité de trouver une solution de vente satisfaisante, les autorités allemandes privilégient la poursuite de la production sous $\mathrm{AP}$, en un temps de pénuries accrues. À la Libération, le groupe est restitué à ses légitimes propriétaires. AN, AJ38 2043-2045. Le Bot (2007). 
Jean Charles-Brun l'inamovible président de la Fédération régionaliste française depuis 1900, ami et inspirateur de Hennessy, ne put, malgré tous ses efforts, influencer le régime. Soulignons une autre des contradictions de Vichy qui proclame sa volonté de restaurer les provinces, de mettre en place des gouverneurs assistés d'une assemblée de représentants des forces vives économiques et spirituelles de la région, et qui ne crée en fait qu'un nouvel échelon en prolongement du pouvoir central (Barral, 1974).

\section{Quelle politique industrielle : uniformisation ou polarisation? (1950-2000)}

\section{L'impératifindustriel}

Les dirigeants de la IV ${ }^{\mathrm{e}}$ République, pour l'essentiel, ne partagent pas la conception de leurs prédécesseurs de la III ${ }^{e}$ République, selon laquelle la stabilité des structures économiques reposant sur un socle de PME serait facteur de stabilité sociale. Ils préfèrent favoriser la production afin d'alimenter la consommation et la prospérité. Dans ce nouveau paradigme, l'État trouve à s'appuyer sur des fédérations patronales au positionnement renouvelé et qui accompagnent une reprise du mouvement de concentration et de rationalisation, revivifié par les missions de productivité dépêchées aux États-Unis. Ainsi, le Comité national de la productivité (CNP) lance en octobre 1951, avec de vrais moyens financiers, un programme d'accroissement de la productivité dans dix-huit usines de chaussures du pays (Boulat, 2006).

À Fougères, entre la fin des années 1940 et le début des années 1960, les disparitions d'entreprises s'accélèrent (une quarantaine de fermetures) et l'emploi régresse (environ 3500 disparitions d'emploi) ${ }^{16}$. Durant les années 1960, malgré 5 nouvelles fermetures, les effectifs globaux marquent une progression (environ 4500 emplois) grâce au dynamisme de quelques établissements. Au $1^{\mathrm{er}}$ janvier 1968, les 6 établissements de plus de cent salariés représentent $84 \%$ des effectifs, dont les deux principaux $57 \%$. Cette concentration s'accompagne de modifications de la production. Les pouvoirs publics, avec le soutien de la Fédération nationale de l'industrie de la chaussure, encouragent cette réorganisation fordiste, présentée comme la solution à tous les problèmes, sans d'ailleurs s'interroger quant aux possibilités de débouchés d'une production bas de gamme qui arrive sur un

16. Archives privées, J.-Y. Trividic, « La transformation des structures et de l'emploi dans l'industrie fougeraise de la chaussure ", Direction régionale du travail et de l’emploi de Bretagne, mars 1980. 
marché déjà fort investi par les grands groupes ${ }^{17}$. Ainsi, les principaux établissements délaissent de plus en plus les produits de moyenne gamme qui ont fait leur réussite et le marché intérieur, soumis aux exigences de la distribution de masse et également à la concurrence italienne, pour la quête de gros contrats sur les marchés extérieurs réclamant des produits bas de gamme. En novembre 1969, la Fédération nationale de l'industrie de la chaussure présente aux pouvoirs publics une demande d'aide à l'exportation et à la concentration des entreprises, lesquelles devaient passer pour l'ensemble du pays de 700 à 200. Cette aide se concrétise en 1970 par le « Plan chaussure ", qui ouvre un crédit de 30 millions de francs afin de permettre aux principaux fabricants d'installer des magasins à l'étranger. La dévaluation du franc en 1969 renforce les positions à l'exportation : en 1972, les deux principales entreprises fougeraises (Réhault et JB Martin) y réalisent $40 \%$ de leur chiffre d'affaires. Cette conquête de marchés extérieurs se fait au profit d'une amélioration de la productivité et aux dépens des établissements les plus petits, impliquant une poursuite de l'hémorragie des emplois : entre janvier 1969 et janvier 1973, 445 emplois sont perdus sur un total d'environ 4000 . La spécificité des districts industriels, les aspects positifs et les points forts de leur héritage sont gommés, pour les faire entrer dans la voie étroite du modèle industriel dominant. Le travail se rationalise, les chaînes de montages se généralisent, la gamme des modèles (et leur qualité) se réduit pour abaisser les coûts ; les tâches sont parcellisées et chronométrées, la productivité et la production s'accroissent. D'une industrie à main-d'œuvre qualifiée, la chaussure fougeraise se transforme en industrie de main-d'œuvre tout court, avec une nette féminisation de l'emploi. Fougères n'est plus un district industriel. Entre la fin des années 1940 et les années 1960, les principales spécificités du système ont fait long feu : disparition d'un grand nombre d'établissements, des possibilités de mobilité pour les salariés, de flexibilité et de coopération pour l'ensemble de la place ; disparition des banques locales et mise en place d'une politique de crédit normalisée ; fin des pratiques locatives de l'USMC. Fougères devient un territoire industrialisé banalisé, avec une forte proportion de salariés dans le secondaire, beaucoup dans la chaussure, mais également dans des usines textiles et électroniques installées là par la déconcentration industrielle ${ }^{18}$.

17. Archives du ministère de l'Économie, des Finances et de l'industrie (CAEF), B57658, Étude sur la reconversion dans les industries de la chaussure, 1948-1954.

18. AN F12, Fond de développement économique et social (FDES), dossiers des entreprises ayant sollicité un prêt ou une prime pour décentralisation, 1954-1962. 
En effet, la politique industrielle française se prolonge à travers une politique d'aménagement du territoire qui conduit l'État, durant les années 1960, à promouvoir une décentralisation industrielle qui n'est en fait qu'une déconcentration des sites de production, sans les directions stratégiques et les bureaux d'étude (Le Bot, Marzin, 2010). Georges Pompidou, premier ministre, puis président de la République, personnifie ce volontarisme en matière d'équipement et de modernisation industrielle (ainsi les " champions nationaux ») (Roussel, 1994). La création de la Délégation à l'Aménagement du Territoire et à l'Action régionale (DATAR) en 1963, concrétise une volonté de piloter l'aménagement sous une forme centralisée, alors que la $I V^{e}$ république avait su faire émerger un partenariat entre acteurs locaux et pouvoir central (Caro et alii, 2002). De ce point de vue, la loi signée en 1972 par Pompidou, fondant la région comme établissement public encadré par un préfet, représente, plutôt qu'une phase de décentralisation, un renforcement de l'État sur l'économie des territoires, à travers un renouvellement des modalités de dialogue entre celui-ci et les élus locaux ${ }^{19}$.

Cette politique applique au territoire la taylorisation usinière : rationalisation et productivité en sont les maîtres mots ; la quête d'une main-d'œuvre bon marché, facilitée par l'exode rural, l'expression. La mobilisation verticale de l'économie, associant à la fois les responsables patronaux et une politique territoriale d'ensemble, produit une politique cohérente, qui toutefois ne tient pas compte des spécificités des territoires et en l'espèce des celles des districts industriels : l'« impératif industriel » qui « visant à homogénéiser les structures industrielles » et « réduisant l'action publique sur un territoire à une projection d'un plan sur un espace inerte [...] a condamné de fait la diversité observé dans les différents SPL » (Courlet, 2006 : 15). Entre 1940 et 1970, un même déclin des districts s'observe au Royaume-Uni, selon des modalités comparables de concentration des entreprises encouragée par l'État à des fins de modernisation industrielle (Zeitlin, 1995) tandis que, comme en France, le processus de centralisation du système bancaire affecte l'investissement local (Carnevali, 2005), l'autonomie des autorités locales se réduit, les syndicats ouvriers reportent leurs actions dans le cadre national ou de branche.

\section{Une voie alternative face à la crise?}

Cette politique, promue par l'État et la représentation patronale, va se heurter à la situation née des bouleversements de la fin des années 1960

19. JORF 9 juillet 1972, loi du 5 juillet 1972, création et organisation des régions. 
et surtout des années 1970-1980, impliquant progressivement une réévaluation de l'action publique. En effet, la crise économique de 1973 met un terme à la période de forte croissance. Le capitalisme mondial engage de profondes mutations, avec une redistribution des activités à l'échelle de la planète, qui, chemin faisant, remettent en cause le modèle fordiste. Les usines à redistribuer sur le territoire deviennent rares. Sur le plan politique, les lois de décentralisation de 1982-1983 offrent aux régions un plus grand rôle dans l'aménagement du territoire, dont la DATAR n'est plus le principal moteur. La combinaison de ces facteurs conduit à réévaluer les potentiels locaux de création d'activités et d'emplois. Les districts industriels paraissent des gisements de croissance potentielle.

Pour autant, les survivances des districts de la chaussure échappent au spectre de l'analyse publique. Des configurations, telles Cholet ou Romans, qui n'ont pas suivi la voie fordiste, mais ont su développer une stratégie de conquête de segments de marchés autour de produits bien définis (le bas de gamme pour la première, le luxe pour la seconde), ont montré dans ce contexte mouvant, une capacité de résistance exemplaire.

Lindustrie de la chaussure choletaise, dans le Maine-et-Loire, peut compter sur la main-d'œuvre abondante des campagnes, libérée de manière saisonnière puis par la modernisation agricole. Elle se spécialise dans un article bon marché, de fabrication grossière et de valeur médiocre. De manière contracyclique, pendant la crise des années 1930, son chiffre d'affaires s'accroît de $35 \%$. Avec les Trente Glorieuses, elle connaît un second décollage : employant plus de 7000 personnes en 1956 pour $12 \%$ de la production nationale ; 16800 pour $25 \%$ de la production dix ans plus tard. Cela se double d'un effort de modernisation et de développement des marchés extérieurs. M. Lescure évoque « une réussite industrielle fondée sur une large mobilisation du territoire »(Lescure, 2006 :412). Malgré l'organisation taylorienne du montage associée à une grande flexibilité de l'emploi, féminisé, pour la phase de piqûre, le développement choletais ne passe ni par l'intégration verticale, ni même par la croissance de la taille des entreprises, mais par essaimage des entreprises. Ainsi, le nombre de PME croît : une centaine en 1930, 180 en 1956, 160 en $1967^{20}$. Le groupe ERAM, constitué en 1946 autour d'une société de fabrication prolongée par des succursales de vente et associée à d'autres entreprises du district au sein d'un groupement industriel, domine l'ensemble. Cholet en plein dynamisme n'a pas à s'inquiéter des recommandations des pouvoirs publics et de la fédération patronale quant au modèle fordiste (d'autant que

20. ABdF, rapports d'inspection de la succursale de Cholet, 1932, 1956, 1967. 
l'opposition à l'État central fait partie de la culture du Choletais), contrairement à Fougères qui reçoit celui-ci comme une bouée de sauvetage. Au début des années 1980, le Choletais compte encore près de 120 entreprises (Courault, 2006). Ce n'est qu'avec les années 2000 que ce tissu est durement frappé par les difficultés (1 200 licenciements en 1999 ; 5700 emplois supprimés entre 1994 et 2004, pour 12700 salariés). L'essentiel des PME a fusionné au début des années 1990 au sein de 4 structures ; sans toutefois de prolongements dans la distribution, ni de stratégie de marque. Ces groupements disparaissent entre 2002 et 2004 face à la concurrence internationale. ERAM (3 000 salariés en 2004) mais aussi les petites entreprises indépendantes, qui bénéficient de ses commandes, ont su résister grâce à un positionnement ancien de marque et dans la distribution.

A Romans, la tendance a été à la réduction du nombre d'entités : 204 entreprises en 1948, 69 en 1969, trois en 2000 (sans compter une dizaine de très petites entreprises) : Jourdan, Kélian et Clergerie, totalisant 1214 emplois (Sauvageon, 2001). En 2009, seule la dernière demeure en tant que fabrique (les deux autres subsistant comme marques à la production délocalisée et, pour l'essentiel, externalisée). Cela s'est accompagné d'une concentration de l'emploi (22 salariés par entreprise en 1948, près de 100 en 1969, plus de 400 dans les trois dominantes en 2000), mais aussi d'une spécialisation dans la chaussure de luxe pour femmes (88\% de la production en 1981). Le maintien, jusque dans les années 1980, d'un niveau d'emploi important (autour de 3000 emplois) résulte largement de ce confinement sur un segment de marché très ciblé, autour d'un produit stylisé, avec une mise en place dans des boutiques destinées à une clientèle aisée, voire pour la haute-couture. Toutefois, la faiblesse de Romans résulte dans une large mesure de sa dépendance aux externalités, notamment financières, contribuant à un faible investissement productif. L'hypothèse pour la fin de la période, est que les investisseurs s'avèrent plus intéressés par les marques et les boutiques que par l'outil de production.

À Fougères, la situation s'est fortement dégradée dès le début des années 1970 (perte du tiers des emplois en 1976). Les entreprises ont perdu le bénéfice (négocié par les pouvoirs publics et la fédération patronale) de contrats d'importations soviétiques pour des chaussures bas de gamme en grandes séries : en 1973-1974, Réhault voit sa production chuter de plus de $43 \%$, alors que celle destinée à l'U.R.S.S. a régressé de $90 \%$; l'entreprise ferme en $1976^{21}$. Au 31 décembre 1984, il ne reste plus que

21. Archives de la CCI de Fougères, dossier Réhault redressement, rapport, 21 janvier 1975. 
10 fabriques occupant 1522 salariés. L'année suivante la dernière importante, JB Martin, connaît un dépôt de bilan. La période 1985-1986 constitue une nouvelle séquence noire pour l'industrie fougeraise dans son entier (une autre s'observe en 1994-1997) qui perd 1500 emplois avec la fermeture d'une quinzaine d'usines. En 1986, JB Martin est relancée par un repreneur sur des bases inédites pour le fougerais, avec la constitution d'un groupe, son expansion (rachat d'entreprises concurrentes, reprise de marques sous licence), son internationalisation commerciale (rachat d'un réseau de distribution en Allemagne, installation de corners et de boutiques en Chine) et productive (délocalisation d'une partie de la production au Maroc et en Chine). Depuis 2009, JB Martin est la dernière fabrique de chaussures de Fougères, avec moins de 200 emplois sur place.

Ainsi, lorsque l'État a été suivi dans ses choix fordistes (Fougères), la situation s'est fortement dégradée dès le début des années 1970, tandis que des voies de développement alternatifs ont permis un maintien plus durable, jusqu'à la fin des années 1990, d'autres territoires industrialisés (Cholet et Romans).

\section{Conclusion}

Il ne reste en 2007 que 114 fabriques de chaussures dans tout le pays, pour une production (36 millions de paires) divisée par 6 en trente ans, selon une baisse continue avec une accélération au cours des années 2000-2005. L'issue de cette histoire peut donc sembler paradoxale. Alors que l'État, à partir des années 1990 et surtout des années 2000 avec la politique des " pôles de compétitivité " (DATAR, 2004), se penche sur les possibilités de développement de territoires dédiés à des activités spécifiques autour d'un système local de compétences, les entreprises et les territoires de la chaussure, parmi d'autres, qui ont suivi cette voie de manière pionnière s'avèrent abandonnés dans le maelström de la recomposition industrielle mondiale. Il est vrai que les "pôles de compétitivité " sont retenus notamment en fonction de caractéristiques innovantes, définies par l'Institut national de la statistique (INSEE) selon un degré significatif d'innovation technologique (mesuré par les dépôts de brevets) et selon des secteurs d'activités : chimie, pharmacie et biotechnologie, nouveaux matériaux et matériaux composites, technologies de l'information et de la communication. Cette définition écarte de fait le secteur de la chaussure, dominé par l'innovation incrémentale, l'innovation d'adaptation et l'innovation 
importée d'autres secteurs, qui sort ainsi des priorités des politiques publiques en matière industrielle ${ }^{22}$.

La dynamique de l'industrie s'appuie sur des ressources (finances, maind'œuvre, énergies, techniques), en même temps que sur un triptyque " produits, processus de production, marchés ». Les mobilisations du temps de guerre, puis de la période fordiste, conduisent à négliger produits et marchés au profit des seuls gains de productivité (les marchés paraissent ouverts à des produits peu élaborés), rendant malaisée l'adaptation aux changements de conjoncture. Les exemples de réussite en phase concurrentielle correspondent à une stricte adéquation entre le produit, le marché cible et le processus de production mis en œuvre. Fougères avant 1930, Romans jusque dans les années 1980, Cholet jusqu'en 2000 répondent à cet impératif.

Des politiques publiques menées en France au XX $\mathrm{XX}^{\mathrm{e}}$ siècle, ont ainsi contribué, dans une certaine mesure, à la disparition des SPL de la chaussure par des choix indifférenciés ne tenant pas compte des spécificités des territoires concernés.

\section{u références}

Bagnasco A., 1977. Tre Italie : la problematica territoriale dello sviluppo italiano, Bologne, Il Mulino.

Barral P., 1974. Idéal et pratique du régionalisme dans le régime de Vichy, Revue française de science politique, 5, 911-939.

Becattini G., 1979. Dal settore industriale al distretto industriale. Al la ricerca dell'unita' d'analisi dell'economia industriale, Rivista di Economia e Politica Industriale, 1, 7-21.

Boulat R., 2006. Jean Fourastié, la productivité et la modernisation de la France (années 1930-1950), thèse d'histoire, université de Franche-Comté, Besançon.

Carnevali F., 2005. Europe's advantage: banks and small firms in Britain, France, Germany, and Italy since 1918, Oxford, Oxford University Press.

22. http://www.insee.fr/ 
Caro P., Dard O., Daumas J.-C. (dir.), 2002. La politique d'aménagement du territoire : racines, logiques et résultats, Rennes, PUR.

Courlet, C., Soulage, B. (éd.), 1995. Industrie, territoire et politique publique, Paris, L'Harmattan.

Courlet C., 2006. Les systèmes productifs localisés en France: une histoire récente, in M. Lescure (dir.), La mobilisation du territoire. Les districts industriels en Europe occidentale du XVIIe au XXe siècles, Paris, CHEFF, 11-27.

Courault B., 2006. PME et industrialisation. Que sont devenues les PME du «miracle choletais» (1945-2004) ?, in M. Lescure, op. cit., 413445.

Datar, 2004. La France, puissance industrielle. Une nouvelle politique industrielle par les territoires. Réseaux d'entreprises, vallées technologiques, pôles de compétitivité, Étude prospective, 1 vol. reprog.

Daumas J.-C., 2007. Districts industriels : du concept à l'histoire. Les termes du débat », Revue économique, Paris, 58(1), 131-152.

Daumas J.-C., Chatriot A., Fraboulet D., Fridenson P., Joly H. (éd.), 2010, Dictionnaire historique des patrons français, Paris, Flammarion.

Druelle-Korn C., 2004. Un laboratoire réformateur : le département du Commerce en France et aux États-Unis de la Grande Guerre aux années vingt, thèse d'histoire, IEP de Paris.

Dubasque F., 2008. Jean Hennessy (1874-1944). Argent et réseaux au service d'une nouvelle république, Rennes, PUR.

Fridenson P., 1987. Un tournant taylorien de la société française (19141918), Annales ESC, 5, 1031-1060.

Godfery J., 1987. Capitalism at War: industrial policy and bureaucracy in France, 1914-1918, Hamburg, Leamington Spa, New-York, Berg.

Guillaume S., Lescure M., 2008. Les PME dans les sociétés contemporaines de 1880 à nos jours. Pouvoir, représentation, action, Bruxelles, Peter Lang.

Horn J. (éd.), 1997. State, society and mobilization in Europe during the First World War, Cambridge, Cambridge University Press.

Joly H. (dir.), 2004. Les Comités d'organisation et l'économie dirigée du régime de Vichy, Caen, CRHQ.

Kuisel R., 1984. Le capitalisme d'État en France: modernisation et dirigisme au XXe siècle, Paris, Gallimard.

Le Bot F., 2005. La «Famille » du cuir contre Bata. Malthusianisme, protectionnisme, xénophobie et antisémitisme dans le monde de la chaussure en France, 1930-1950, Revue d'histoire moderne et contemporaine, 4, 131-151.

Le Bot F., 2007. La fabrique réactionnaire. Antisémitisme, corporatisme et spoliations dans le cuir, 1930-1950, Paris, Presses de Sciences Po. 
Le Bot F., Marzin F., 2010. Le mai 1968 breton et ses acteurs face à une révolution pompidolienne dans l'économie des territoires, M. Margaizar, D. Tartakowsky (dir.), 1968, entre libération et libéralisation. La grande bifurcation, Rennes, PUR, 237-258.

Le Bot F., Perrin C., 2011. Des historiographies en perspective : PME, territoires et industrialisation en Espagne et en France, F. Le Bot, C. Perrin (dir.), Les chemins de l'industrialisation en Espagne et en France. Les $P M E$ et le développement des territoires, (XVIII ${ }^{e}-X X I^{e}$ siècles), Bruxelles, Peter Lang éd., 20-71.

Le Galès P., Trigilia C., Voelzkow H., 2001. Local production systems in Europe: rise or demise? Oxford, Oxford University Press.

Le Galès P., Trigilia C., Voelzkow H., 2004. Changing governance of local economies: Reponses of European local production systems, Oxford, Oxford University Press.

Le Galès P., 2005. Gouvernance des économies locales en France : à la recherche de la coordination perdue, L'année de la régulation. Economie, institutions, pouvoirs, 8, Presses de Sciences po, 105-135.

Lescure M., 2006. Les territoires du choletais (1900-1960). Cultures politiques et systèmes productifs, in M. Lescure, op. cit., 393-412.

Marshall A., 1906. Principes d'économie politique, Paris (1 ${ }^{\text {ère }}$ éd. Londres, 1890).

Odaka K., Sawai M. (ed.), 1999. Small firms, large concerns: the development of small business in comparative perspective, Oxford, Oxford University press.

Ozouf M., 2009. Composition française. Retour sur une enfance bretonne, Paris, Gallimard.

Perrin C., 2007. Entre glorification et abandon. L'État et les artisans en France (1938-1970), Paris, CHEFF.

Perrin C., 2008. Le travail dans l'imagerie de la Révolution nationale, Gavroche, 155, 22-29.

Polanyi K., 1983. La Grande Transformation, Paris, Gallimard (1 $1^{\text {ère }}$ éd. 1944).

Roussel E., 1994. Georges Pompidou, 1911-1974, Paris, JC Lattès.

Sauvageon J. (dir.), 2001. Les Romanais, Romans et la chaussure, 150 ans d'histoire, Valence, ACCES et Édition du Peuple Libre et Notre Temps.

Sick K., 2003. Deux formes de synthèse sociale en crise. Les classes moyennes patronales de la Troisième République à la lumière d'une comparaison franco-allemande, Revue d'histoire moderne et contemporaine, 4, 135-154.

Wachter S., 1989. Politiques publiques et territoires, Paris, L'Harmattan. 
Zalio P.-P., 2004. Territoires et activités économiques. Une approche par la sociologie des entrepreneurs, Genèses, 56, 4-27.

Zalio P.-P., 2005. The Mobilisation of Local Resources for an urban economic project in the European context, in R. Salais, R. Villeneuve (eds.), Europe and the politics of capabilities, Cambridge, Cambridge University Press, 91-108.

Zalio P.-P., 2006. Marseille 1880-1960. Pourquoi peut-on parler de district industriel ?, M. Lescure, op. cit., 181-196.

Zarca B., 1986. L'artisanat français. Du métier traditionnel au groupe social, Paris, Économica.

Zdatny S., 1999. Les artisans en France au XXe siècle, Paris, Belin.

Zeitlin J., 1995. Why Are There no Industrial Districts in the United Kingdom?, A. Bagnasco, C. Sabel (éd.): Small firms in Europe, London, Pinter, p. 98-114.

Zeitlin J., 2007. Industrial Districts and Regional Clusters, G. Jones, J. Zeitlin (éd.), The Oxford Handbook of Business History, Oxford University Press, 219-243.

Cédric Perrin (cp2002@orange.fr) Chercheur associé, IDHE Université Paris Ouest Nanterre La Défense 